\title{
Editorial
}

\section{Special Issue on Catalyst Deactivation and Regeneration}

\author{
Pedro Castaño ${ }^{1,2}$ (D)
}

Citation: Castaño, P. Special Issue on Catalyst Deactivation and Regeneration. Catalysts 2021, 11, 798. https: / / doi.org/10.3390/catal 11070798

Received: 13 June 2021

Accepted: 29 June 2021

Published: 30 June 2021

Publisher's Note: MDPI stays neutral with regard to jurisdictional claims in published maps and institutional affiliations.

Copyright: (c) 2021 by the author Licensee MDPI, Basel, Switzerland. This article is an open access article distributed under the terms and conditions of the Creative Commons Attribution (CC BY) license (https:// creativecommons.org/licenses/by/ $4.0 /)$.
1 Department of Chemical Engineering, University of the Basque Country (UPV/EHU), 48080 Bilbao, Spain; pedro.castano@ehu.eus or pedro.castano@kaust.edu.sa

2 Multiscale Reaction Engineering, KAUST Catalysis Center, King Abdullah University of Science and Technology (KAUST), Thuwal 23955-6900, Saudi Arabia

Catalyst deactivation causes major losses in the chemical industry worldwide every year. The deactivation phenomenon does not only drop the catalytic activity, but forces an allocation of time and a regeneration of resources, which, at the same time, involves wasting more feedstock and materials, and emitting more $\mathrm{CO}_{2}$. Catalyst deactivation can occur by means of metal sintering, phase transformation, thermal degradation, and coke deposition. Regeneration per-se is a costly process and is detrimental for the carbon balance that can potentially, and irreversibly, harm the valued catalysts. The limitless chase of better catalysts with enhanced properties elevates the trouble even more, as both deactivation and regeneration are more challenging.

To complicate things, the study of deactivation at the laboratory scale is not straightforward in continuous reactors because it involves incorporating one more variable: time. Besides, it is not easy in batch systems either because both reaction and deactivation evolve simultaneously, making it difficult to decouple these two phenomena. These hurdles that ultimately become pitfalls have been elaborated in detail in previous and highly recommended works. This special issue gives an overview of the state-of-the-art catalytic deactivation and regeneration in several relevant catalytic processes such as hydrotreating or cracking, among others. As it can be seen in the works included in this special issue together with the other works published in Catalysts and other related journals, there is a strong need to embrace the deactivation and regeneration aspects from a transversal and multidisciplinary point of view, and to merge concepts of material science, reaction engineering, spectroscopy, among others. Catalysts journal is at the epicenter of the field of deactivation and regeneration and, as it can be seen in Figure 1, the number of articles keeps increasing.

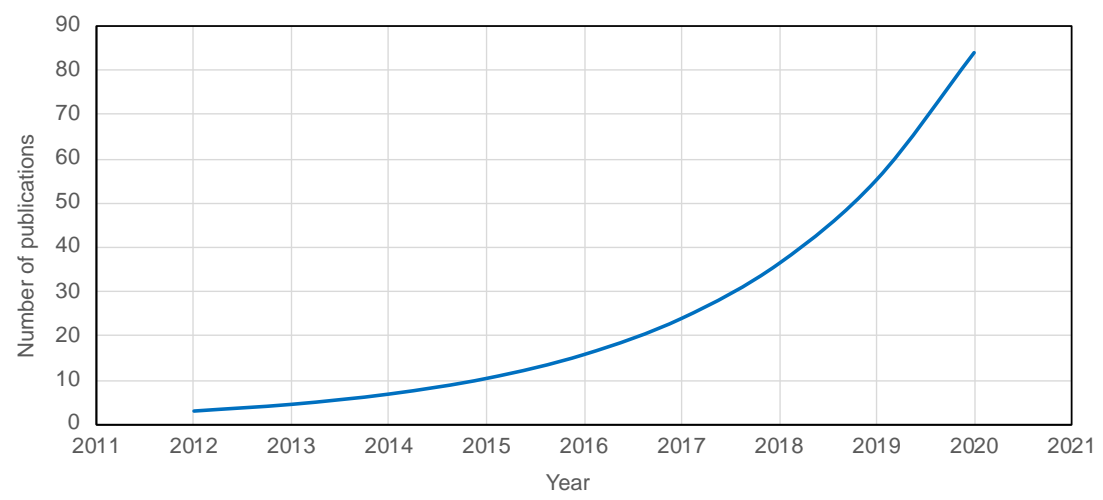

Figure 1. Number of publications in Catalysts journal in the period 2012-2020, using "deactivation" or "regeneration" search terms, according to Scopus ${ }^{\mathrm{TM}}$.

Alkhlel and de Lasa [1] have studied the effect of catalyst-to-oil ratio on the coke formation (among other effects) during the cracking of 1,3,5-tri-isopropyl benzene under 
relevant fluid catalytic cracking (FCC) conditions. These authors have shown that the highest values of catalyst-to-oil ratio involve more chances for bimolecular reactions and, as a result, enhanced coke formation. This kind of result can lead to further optimization of process parameters and design to control the coke formation.

The instabilities and transient state happenings in catalytic processes, particularly at the beginning of the reaction, are valuable information from the kinetic point of view. In line with this, Cao et al. [2] have used these transient data to accelerate kinetic modeling discovery and fitting. This interesting avenue calls for a reconsideration of the features that make the selection of the catalysts for a given process, such as the one selected: hydrotreating.

Generally, more sophisticated catalyst with enhanced activity and selectivity normally means that it is more "delicate". This rule leads to innovation in the regeneration step too. Soutelo-Maria et al. [3] have investigated solvent wash, hydrogen treatment, and sonication to regenerate Raney ${ }^{\circledR}$-Nickel catalyst for the synthesis of high-valued aminoester monomers. Their results show that hydrogen treatment is the best strategy to recover the full original catalytic activity.

When deactivation is due to coke fouling, the most standardized methodology to regenerate the catalysts is combustion-calcination. However, this strategy is sometimes difficult to control and it is risky for the most sophisticated catalytic materials. In this framework, Astafan et al. [4] have employed a fixed bed dielectric barrier reactor with non-thermal plasma (NTP) waves to regenerate different zeolite frameworks (MFI, MOR, and FAU). These authors discovered that the efficiency of coke oxidation by non-thermal plasma is very sensitive to the coke nature, which is also linked at the same time with the pore topology of the zeolite. Compared to classical calcination with air, non-thermal plasma is a much cleaner technology. Srour et al. [5] followed a relatively similar strategy for spent hydrotreating-hydrodesulfurization catalysts using dielectric barrier discharge (DBD) non-thermal plasma. Their results show that, for this process, certain parameters require further studies as the catalyst is not completely regenerated at the expense of forming other interesting species on the surface of the catalyst.

As an overview, the works presented in this special issue assure a bright future for the field of catalyst deactivation and regeneration, with many open questions and chances for catalyst and process innovations.

Conflicts of Interest: The author declares no conflict of interest.

\section{References}

1. Alkhlel, A.; de Lasa, H. Catalyst/Feedstock Ratio Effect on FCC Using Different Catalysts Samples. Catalysts 2019, 9, 542. [CrossRef]

2. Cao, N.Y.P.; Celse, B.; Guillaume, D.; Guibard, I.; Thybaut, J.W. Accelerating Kinetic Parameter Identification by Extracting Information from Transient Data: A Hydroprocessing Study Case. Catalysts 2020, 10, 361. [CrossRef]

3. Soutelo Maria, A.; Dubois, J.L.; Couturier, J.L.; Brebion, M.; Cravotto, G. Regeneration of Raney ${ }^{\circledR}$-Nickel Catalyst for the Synthesis of High-Value Amino-Ester Renewable Monomers. Catalysts 2020, 10, 229. [CrossRef]

4. Astafan, A.; Sachse, A.; Batiot-Dupeyrat, C.; Pinard, L. Impact of the Framework Type on the Regeneration of Coked Zeolites by Non-Thermal Plasma in a Fixed Bed Dielectric Barrier Reactor. Catalysts 2019, 9, 985. [CrossRef]

5. Srour, H.; Alnaboulsi, A.; Astafan, A.; Devers, E.; Toufaily, J.; Hamieh, T.; Pinard, L.; Batiot-Dupeyrat, C. Elimination of Coke in an Aged Hydrotreating Catalyst via a Non-Thermal Plasma Process: Comparison with a Coked Zeolite. Catalysts 2019, 9, 783. [CrossRef] 